\title{
Diagnóstico de problemas de manejo del suelo con abonos orgánicos utilizando un minisimulador de lluvia
}

\author{
Diagnostics of soil management problems with organic fertilizers using \\ a miniature rain simulator
}

\author{
Jairo Sarasty B. ${ }^{1}$; Jimmy Andrés Ortega M. ${ }^{2}$; Jesús Antonio Castillo F. ; Germán Chaves Jurado. \\ ${ }^{1}$ Ingeniero Agroforestal. Universidad de Nariño, San Juan de Pasto, Colombia, jsarasty14@gmail.com. \\ ${ }^{2}$ Ingeniero Agroforestal. Universidad de Nariño, San Juan de Pasto, Colombia, jhiandres@gmail.com. \\ ${ }^{3}$ Profesor Asociado. Ph.D. Universidad de Nariño, San Juan de Pasto, Colombia, jacf1995@gmail.com. \\ ${ }^{4}$ Profesor. M.Sc. Universidad de Nariño, San Juan de Pasto, Colombia, g-ch-j@hotmail.com.
}

Citar: Sarasty, J.; Ortega, J.; Castillo, J.; Chaves, G. 2017. Diagnóstico de problemas de manejo del suelo con abonos orgánicos utilizando un minisimulador de lluvia. Rev. Cienc. Agr. 34(2): 46 - 61. doi: http://dx.doi.org/10.22267/ rcia.173402.71.

Recibido: Junio 13 de 2016.

Aceptado: Diciembre 07 de 2016.

\section{RESUMEN}

El estudio se realizó en zona de ladera en Nariño, Colombia, en un suelo Pachic Fulvudands, bajo un sistema productivo de cebolla de rama Allium fistulosum L., sometido a aplicaciones con abonos orgánicos por más de diez años; se utilizó un Diseño Irrestrictamente al Azar en arreglo trifactorial $(3 \times 4 \times 6)$, donde el primer factor correspondió a tres pendientes de terreno $\left(10^{\circ}, 15^{\circ}\right.$ y $\left.25^{\circ}\right)$, el segundo factor a los usos del suelo con cultivo de cebolla, bajo aplicación de tres abonos orgánicos (U1: Gallinaza; U2: Bovinaza; U3: Residuos vegetales) y un testigo U4 (Área en conservación con árboles) y el tercer factor correspondió a tiempos de simulación de lluvia $(5,10,15,20,25$ y 30 min). Se aplicó una intensidad de lluvia de $15,5 \mathrm{~mm} / \mathrm{h}$, como resultado de la calibración del minisimulador de lluvia según datos de precipitaciones de la zona. Los resultados mostraron que el uso de suelo con bovinaza U2, presentó valores más altos para lámina de escorrentía $(1,01 ; 2,51$ y 4,96 $\mathrm{mm}$ ) para las tres pendientes, sugiriendo mayor remoción de las partículas del suelo. Para Lámina de Infiltración, los valores más altos se obtuvieron en U4, con valores de 7,18; 6,85 y 6,97 mm, a diferencia de U2 que presentó los valores promedios más bajos y las mayores pérdidas de suelo con 
0,072, 0,284 y 0,595 t.ha-1 para las pendientes evaluadas. Los resultados sugieren, que adición de enmiendas orgánicas acompañados de manejos inadecuados en el largo plazo, no son suficientes para disminuir riesgos por degradación física del suelo.

Palabras clave: degradación, infiltración, escorrentía, estabilidad estructural, andisol.

\begin{abstract}
The study was conducted in the hillside area of the departamento de Nariño, Colombia, in a Pachic Fulvudands soil, under an Allium fistulosum L. crop with organic fertilizers for more than ten years; a completely randomized design in a trifactorial arrangement $(3 \times 4 \times 6)$ was used, where the first factor corresponded to three terrain slopes $\left(10^{\circ}, 15^{\circ}\right.$, and $\left.25^{\circ}\right)$, the second factor to the land uses with Allium fistulosum L. crop, under the application of three organic fertilizers (U1: Hen manure, U2: Bovine manure, U3: vegetable Residues) and a control U4 (Area in conservation with trees): the third factor corresponds to the simulation times by using a rain minisimulator $(5,10,15,20,25$ and $30 \mathrm{~min})$. Throughout the experiment, a rainfall intensity of $15.5 \mathrm{~mm} / \mathrm{h}$ was applied as a result of the calibration according to the daily rainfall data from the zone. The results showed that the soil with bovine manure U2 had the highest values for runoff sheet $(1.01,2.51$ and $4.96 \mathrm{~mm})$ for the three slopes suggesting higher removal of surface particles from the soil. For the infiltration sheet, the highest values correspond to $U 4$, with $7.18,6.85$, and $6.97 \mathrm{~mm}$, respectively, unlike $\mathrm{U} 2$ which presented the lowest average values and the highest soil losses with $0.072,0.284$, and 0.955 t.ha $^{-1}$ in the three slopes. The results suggest that the addition of organic amendments together with soil management, in the long run, are not enough to reduce risks due to physical soil degradation.
\end{abstract}

Keywords: degradation, infiltration, runoff, structural stability, andisol.

\section{INTRODUCCIÓN}

La erosión hídrica es considerada como el mayor proceso de perdida de suelo cultivable en el mundo y se estima que es de 10 millones ha por año (Pimentel, 2006), según Oldeman et al. (1990), señala que la pérdida de suelo por erosión hídrica a nivel global, es el problema más grave de degradación, que tiene que enfrentar la sociedad moderna. Para Fraume, 2007 la erosión del suelo se refiere al deterioro y eliminación del suelo superficial por la acción del agua de escorrentía.
De acuerdo con los primeros estudios realizados por IGAC (1995), entre el 48 y 49\% del territorio colombiano, está afectado por erosión hídrica, el 22,9\% por movimientos en masas y solamente el $24 \%$ no tiene problemas. Otro de los estudios sobre la erosión de las tierras colombianas es presentado por Mendivelso et at (1998), donde reporta que el 83\% de la zona andina colombiana presentan algún grado de erosión. Los datos más recientes de erosión en Colombia los presenta el IDEAM, 2012 y expresa que 52.877.562 ha, están afectadas por algún grado de erosión, 
información que está en proceso de actualización hoy en día.

Un aspecto físico del suelo muy importante lo constituye la estabilidad de sus agregados, lo cual, hace referencia a que éstos, en algunos suelos no soportan por mucho tiempo los efectos de la caída de lluvia y las labores de labranza sin disgregarse, mientras que otros por el contrario permanecen firmes (Amézquita, 1994). En este sentido, Sánchez et al. (2012) manifiestan que la función de la labranza como un sistema conservacionista que integra la preparación de los suelos, genera en los suelos, condiciones físicas adecuadas para el buen flujo del agua y el aire, evitando que se formen en el suelo capas duras que limitan la penetración y crecimiento de las raíces.

Así, una reducción de la erosión hídrica, contribuye a mejorar o mantener la productividad de los suelos y así ayudar a la sostenibilidad de éste recurso, Alvarado et al. (2011), mostraron en su estudio que Phalaris $s p$ presento la mayor efectividad como alternativa para la conservación de suelos en cultivos de papa en zona de ladera, de ahí la importancia de evitar los procesos erosivos mediante la adopción de prácticas de conservación de suelos (Quiroz e Hincapié, 2007), dado que si no se disminuye éste proceso, la capacidad productiva de los suelos erosionados será menor que la capacidad de los no erosionados, debido a que la erosión reduce la fertilidad y la capacidad de almacenamiento de agua en el suelo, por lo tanto "perder suelo es perder agua". Cuando se pierde la estructura, se reduce la tasa de infiltración, la escorrentía aumenta y la capacidad de almacenamiento de agua disminuye (Lal, 1998).

Son numerosos los autores que han trabajado en la relación lluvia - escurrimiento. Y la mejor correlación que se obtuvo fue al relacionar la lámina con intensidad de la lluvia. Al respecto, Rivera-Ruiz et al. (2012) Observó, que es muy importante considerar la humedad del suelo al momento que ocurra el evento y en este sentido el autor expresa que los suelos andisoles tienen alta capacidad de infiltración cuando secos, pero una vez húmedos cambia el comportamiento. El impacto de gotas de lluvia inicia el proceso de mojado del suelo y luego salpicado y remoción de partículas del suelo, finalmente la erosión es una función de la capacidad del flujo superficial para desprender el sedimento (Flanagan et al., 1995; Figueroa et al., 1991) Los sistemas agroforestales por su capacidad de cobertura reducen el efecto de la energía cinética de las lluvias y esto representa disminución en pérdida de suelo. Un dosel vegetal alto y denso puede generar resultados críticos del efecto de las lluvias comparado a un dosel bajo y de capas que reduce el efecto de las gotas de lluvia, Beer et al. (1998).

Mena et al. (2011) en estudios realizados utilizando un minisimulador de lluvia, trabajaron en la susceptibilidad a la erosión hídrica de un Andisol en ladera del departamento de Nariño, encontraron que el uso y manejo de los suelos cultivados con papa y pastos, pueden sufrir erosiones de 0,39 y 0,27 t.ha $^{-1}$, respectivamente, mientras que el bosque, presentó pérdidas por debajo de 0,05 t.ha ${ }^{-1}$.

La estructura del suelo, es el principal factor que condiciona la productividad y fertilidad de los suelos. Debido a la importancia que tienen los procesos de degradación de los recursos naturales y los procesos erosivos de los suelos en la zona andina del departamento de Nariño, surge la necesidad de evaluar los sistemas productivos tradicionales y su estabilidad estructural. 
Para estos estudios, se utilizó un minisimulador de lluvia portátil (Cobo, 1998), además de la realización de algunas pruebas físicas y químicas en laboratorio complementarias, con el fin de apoyar los resultados, el uso de tecnologías que permitan identificar y diagnosticar problemáticas relacionados con el uso y manejo de los suelos.

Con base en lo anterior, el objetivo de éste trabajo, fue diagnosticar problemas de manejo de un suelo Andisol, en un sistema productivo tradicional con Cebolla de rama (Allium fistulosum L.) con abonos orgánicos y en áreas bajo un sistema de conservación con árboles, utilizando un minisimulador de lluvia portátil, teniendo en cuenta tres tipos de pendientes.

\section{MATERIALES Y MÉTODOS}

Este estudio se realizó en el corregimiento de Buesaquillo, Municipio de Pasto, departamento de Nariño, Colombia. A una altura de $2865 \mathrm{msnm}$, con $1^{\circ} 13^{\prime} 33.9^{\prime \prime} \mathrm{LN}$ y $77^{\circ} 13^{\prime} 56.8^{\prime}$ LO, con temperaturas entre $12^{\circ}$ y $18^{\circ} \mathrm{C}$, precipitación media anual de $901,3 \mathrm{~mm}$ y una humedad relativa del $75 \%$. Ecológicamente, la zona donde se llevó a cabo el trabajo, se clasifica como bosque seco montano bajo (Bs-mb), según la clasificación de Holdridge (2000), (IDEAM, 2010).

Topografía y pendiente. En la zona, es característico encontrar un tipo de paisaje de altiplanicie, con un de relieve en lomas (AB), desde muy inclinados a ligeramente escarpados, con pendientes entre 12 y 50\%, rectilíneas, con cimas subagudas y fuertemente disectadas, configurando valles profundos en $\mathrm{V}$, con una intensa actividad erosiva, provocada por las corrientes de agua y el consecuente desgaste de los taludes (IGAC, 2004).

\section{Características físicas y químicas del sue-} lo. Los suelos de ésta zona, corresponden a una consociación Pachic Fulvudands (perfil Ns-13). Son suelos que ocupan la posición de laderas de las mesas, dentro del paisaje de altiplanicie, profundos, bien drenados y de textura franco arcillo arenosa (F-Ar-A) y estructura granular gruesa. Se han desarrollado a partir de cenizas volcánicas, que yacen sobre tobas de ceniza y lapilli. Son suelos moderadamente ácidos, de alta capacidad de intercambio catiónico, alta saturación de bases, altos contenidos de carbono orgánico, medianos contenidos de calcio y magnesio, alto de potasio, bajos en fósforo y de fertilidad alta (IGAC, 2004).

Área experimental. La zona de estudio correspondió a la microcuenca el Quinche, vereda la Alianza, una de las mayores productoras de Cebolla de rama (Allium fistulosum L.) de la región, donde los agricultores durante décadas, han utilizado enmiendas orgánicas como la gallinaza, bovinaza y residuos vegetales.

Los lotes con cultivo de Cebolla de rama se ubican sobre pendientes uniformes entre 7 y $31^{\circ}$, con áreas de conservación con árboles mayores a 10 años.

Diseño Experimental. se utilizó un Diseño Irrestrictamente al Azar en arreglo trifactorial (3x4x6), donde el primer factor correspondió a tres pendientes de terreno $\left(10^{\circ}, 15^{\circ}\right.$ y $\left.25^{\circ}\right)$, el segundo factor a los usos de suelo con cultivo de cebolla de rama Allium fistulosum L., bajo aplicación de tres abonos orgánicos (U1: Gallinaza; U2: Bovinaza; U3: Residuos vegetales) y un testigo U4 (Área en conservación con árboles) (Tabla 1); el tercer factor corresponde a los tiempos de simulación mediante el uso de un minisimulador de lluvia $(5,10$, $15,20,25$ y $30 \mathrm{~min}$ ). 
Tabla 1. Descripción de los usos para diagnosticar problemas de manejo del suelo en un sistema productivo y plantación de árboles mediante la utilización de un minisimulador de lluvia.

\begin{tabular}{cl}
\hline Usos & \multicolumn{1}{c}{ Descripción } \\
\hline U1 & $\begin{array}{l}\text { Lote con cultivo de cebolla de rama }(A \text {. fistulosum L.), con aplicación de abono } \\
\text { orgánico de gallinaza y un historial de manejo mayor a 10 años. }\end{array}$ \\
\hline U2 & $\begin{array}{l}\text { Lote con cultivo de cebolla de rama }(A \text {. fistulosum L.), con aplicación de abono } \\
\text { orgánico de bovinaza y un historial de manejo mayor a 10 años. }\end{array}$ \\
\hline U3 & $\begin{array}{l}\text { Lote con cultivo de cebolla de rama (A. fistulosum L.), con aplicación de abono } \\
\text { orgánico de residuos vegetales caseros y un historial de manejo mayor a 10 } \\
\text { años. }\end{array}$ \\
\hline U4 & Área de conservación con árboles. \\
\hline
\end{tabular}

En todo el experimento se aplicó una intensidad de lluvia de $15,5 \mathrm{~mm} / \mathrm{h}$, como resultado de la calibración según los datos de precipitaciones diarias de la estación Botana desde el año 2000 (IDEAM, 2010).

Minisimulador de lluvia - generalidades. Dadas las limitaciones climatológicas y topográficas de la zona de estudio para realizar las simulaciones en campo, diseñaron ajustes complementarios y estructurales al equipo básico. En ese sentido, se utilizó como herramienta fundamental, el minisimulador de lluvia portátil CIAT (Cobo, 1998), para determinar las relaciones existentes entre suelo erodado, lámina de escorrentía y lámina de infiltración. Para la calibración del minisimulador de lluvia, se utilizó el pluviógrafo marca Eijkelkamp modelo 6987 ZG de registro diario y datos suministrados por el IDEAM para determinar la columna de agua que se ajustó a las condiciones pluviométricas de la zona.

Se diseñó una base recibidora con pendiente graduable, constituida por una estructura en ángulo de hierro de 1/8" de espesor, por ocho piezas las cuales están soldadas entre sí; cuatro de ellas forman la base del recibidor de 65 por $65 \mathrm{~cm}$ (Figura 1A). Dos piezas soldadas en la parte frontal, que originan un ángulo de $90^{\circ}$ con la base, las cuales miden $30 \mathrm{~cm}$ cada una. Por último, las dos piezas de $65 \mathrm{~cm}$ están soldadas con una inclinación de $30^{\circ}$ sobre la base en la parte trasera (Figura 1B).

Estas cuatro piezas soldadas sobre la base, poseen unos orificios a los $10 \mathrm{~cm}$ de altura, por los que pasan dos varillas de acero corrugado de $3 / 8$ ", las cuales, son la base de soporte para el tomador de muestras (monolitos), formando una inclinación de $0^{\circ}$. La varilla frontal es fija y la otra, junto con los dos ángulos traseros, son los que proporcionan la pendiente requerida. Estos poseen unos orificios sobre los cuales se puede rotar la varilla de soporte cada $5^{\circ}$ hasta los $45^{\circ}(100 \%)$ de pendiente (Figura 1C). 


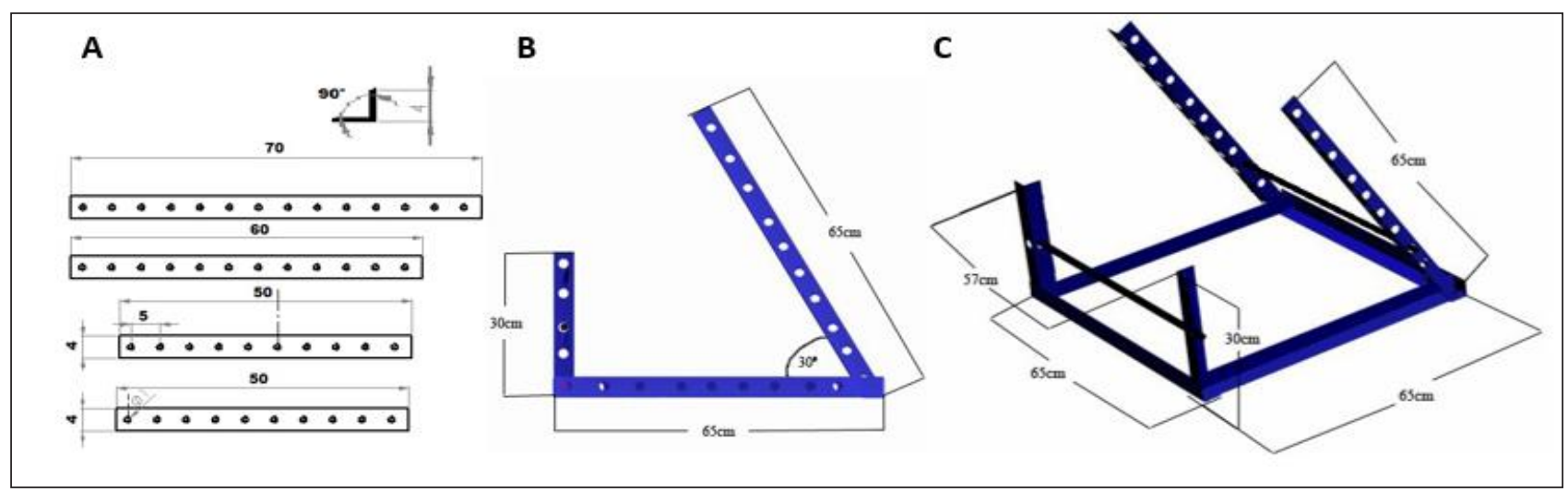

(A. Diseño de 8 piezas base recibidora; B. Piezas laterales soldadas y formando un ángulo de $90^{\circ}$ y $30^{\circ}$; C. Base recibidora con orificios de soporte cada $5^{\circ}$ hasta los $45^{\circ}$ para un $100 \%$ de pendiente).

Figura 1. Piezas del diseño de base recibidora de muestras de suelo.

Tomador de muestras (monolitos). El muestreador (Figura 2), está formado por cuatro partes. Un cajón desarmable con dos partes laterales de $53 \times 17 \mathrm{~cm}$ de alto, una frontal de 43 x 15cm y una trasera de $43 \mathrm{x}$ $17 \mathrm{~cm}$. Estas dos últimas, poseen una ceja de cada lado de $2,5 \mathrm{~cm}$, sobre las cuales van sujetos tres tornillos de acero inoxidable en cada esquina, para sujetarse a las otras dos piezas y dar el soporte desarmable del cajón. Sobre las dos piezas laterales, está soldado un riel a $2 \mathrm{~cm}$ de altura sobre la base, el cual sirve de guía para la lámina de corte. La base de estas cuatro piezas, tienen un filo biselado que permite clavarla en el suelo para sacar el mo- nolito. La lámina cortadora tiene unas dimensiones de 43 x 55cm y está fabricada en acero inoxidable calibre 3/16" tipo 304, siendo muy resistente a impactos.

Muestreo de suelo en campo. Luego de introducir el tomador de muestras en el suelo (Figura 3) por completo y de haber retirado el suelo que queda en la parte externa frontal y lateral del muestreador, se procedió a introducir frontalmente la lámina cortadora, deslizándola por el riel interno hasta cortar y sacar por completo el monolito, luego cada uno es transportado hasta el lugar donde se encuentra el minisimulador de lluvia junto con el recibidor.
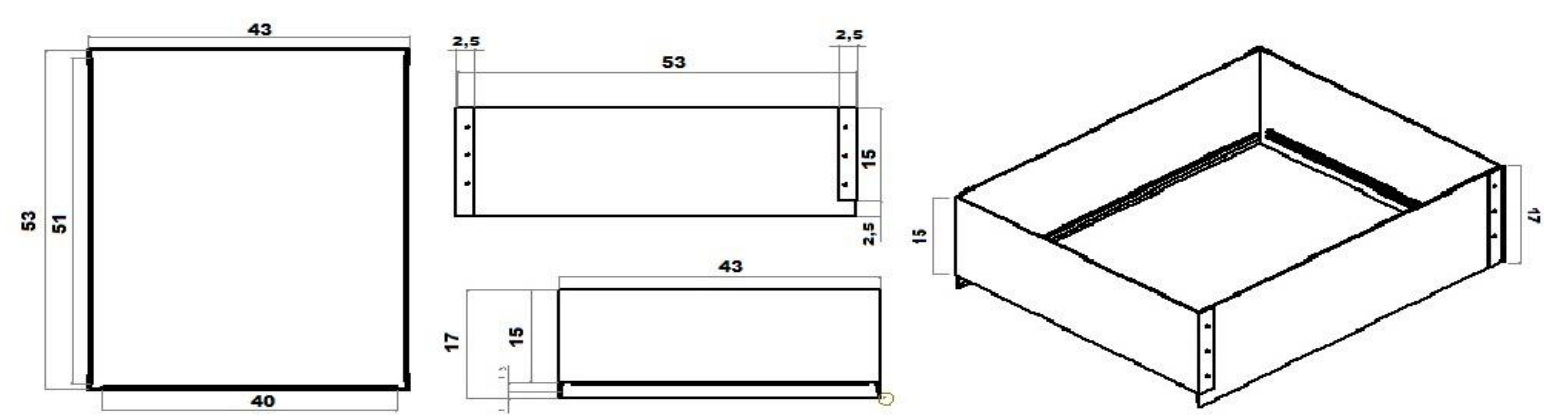

Figura 2. Piezas del diseño del tomador de muestras de suelo. 


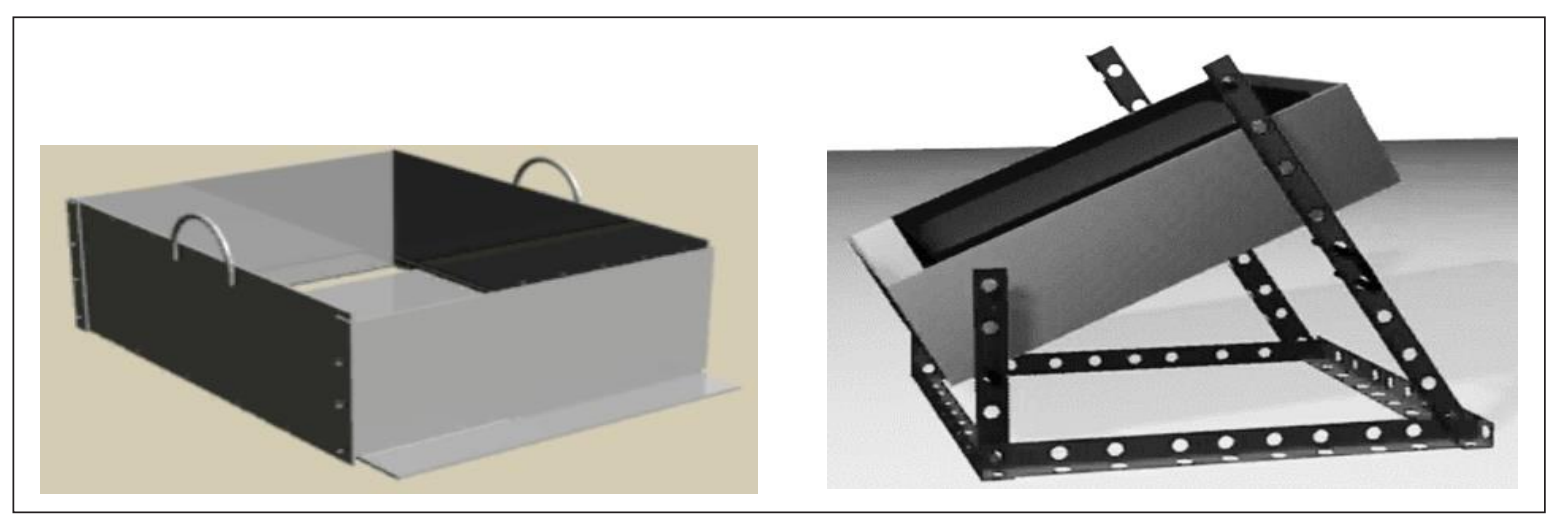

Figura 3. Tomador de muestras de suelo y base recibidora en 3D.

Una vez tomado el monolito, se clavó la bandeja de escorrentía y la bandeja recolectora de excesos, se colocaron sobre la base recibidora (Figura 3). Al tener la muestra sobre la base recibidora, ésta se ajustó a la pendiente deseada, se fijó el trípode del minisimulador para que la lluvia cayera sobre el área efectiva de la bandeja de escorrentía, luego se procedió a realizar las simulaciones.

Para cada tiempo de lluvia simulada, cada cinco minutos, se recogieron los volúmenes de la bandeja de escorrentía y la bandeja de excesos, se midió en probetas y luego se trasvasaron a frascos de vidrio previamente rotulados y pesados. Estos fueron llevados al laboratorio para secarlos al horno a $105^{\circ} \mathrm{C}$ por 24 horas, con el fin de tomar su peso y cuantificar la cantidad de suelo erodado por escorrentía para cada uso a las diferentes pendientes. Para calcular el porcentaje de humedad, se tomaron muestras de suelo antes y después de cada simulación, luego se tomaron $25 \mathrm{~g}$ de suelo, se pesaron y se llevaron al horno por 24 horas a una temperatura de $\pm 105^{\circ} \mathrm{C}$ y se calculó la humedad gravimétrica del mismo. Dentro de cada uso de suelo, se delimitaron tres parcelas, cuya dimensión fue de $4 \mathrm{~m}$ de ancho x $12 \mathrm{~m}$ de largo (Figura 4), cada parcela de estas estuvo dividida en tercios así: ter- cio superior, medio e inferior. En cada tercio se tomó un monolito con el muestreador de $43 \times 54 \mathrm{~cm}$ y $15 \mathrm{~cm}$ de alto, para un total de nueve monolitos por cada uso de suelo. Los monolitos de cada parcela del terreno, fueron utilizados para realizar simulaciones a tres pendientes diferentes $\left(10,15\right.$ y $\left.25^{\circ}\right)$.

Análisis de Datos. Un Análisis de Varianza (ANDEVA) fue suficiente para detectar diferencias entre los factores y la interacción de los mismos. Cuando se presentaron diferencias se utilizó la Prueba de Diferencias Mínimas Significativas de Fisher (LSD) para la separación de promedios con una significancia del 95\%, los datos fueron analizados mediante el programa SAS v.9.4, aplicando el siguiente modelo matemático:

$$
Y_{i j k l}=\mu+A_{i}+B_{j}+C_{k}+(A B)_{i j}+(A C)_{i k}+(B C)_{j k}+(A B C)_{i j k}+\epsilon_{i j k l}
$$

Dónde:

$\mathrm{Y}_{\mathrm{ijk} \mathrm{l}}=$ Variable de respuesta

$\mu=$ Efecto de la media

$\mathrm{e}_{\mathrm{ijk \textrm {k }}}=$ Error experimental

$A i, B j, C k=$ factores

$(A B)_{i j^{\prime}}(A C)_{i k^{k}}(B C)_{j k^{\prime}}(A B C)_{i j k}=$ interacciones 


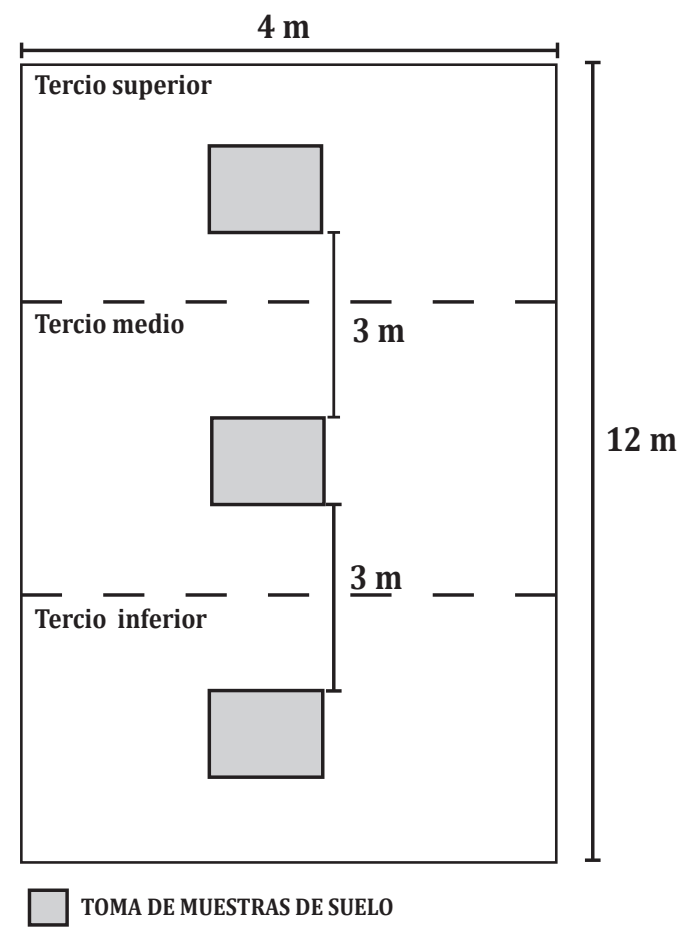

Figura 4. Distribución de los tratamientos en campo.

\section{RESULTADOS Y DISCUSIÓN}

El ANDEVA (Tabla 2) para las variables evaluadas, lámina de escorrentía (LE), lámina de infiltración (LI) y suelo erodado (SE), mostraron significancia estadística para la interacción pendiente por tiempo. Estos factores están influyendo directamente sobre los usos del suelo con abonos orgánicos, gallinaza (U1), bovinaza (U2), residuos vegetales (U3) y área de conservación con árboles (U4). Se observó que a mayor grado de pendiente y a medida que el tiempo de simulación transcurre, estos usos de suelo, se vieron influenciados por el efecto de la lluvia simulada. Igualmente, se observó que los usos de suelo con abonos orgánicos, presentaron diferencias altamente significativas en todas las variables evaluadas. El extenso tiempo de uso, explica el comportamiento de los suelos bajo lluvia simulada. Aunque existe una jerarquía por los materiales orgánicos que controlan la estabilidad de los agregados, según Oades y Waters (1991) se deben examinar los tamaños más afectados.

Tabla 2. Cuadrado medio de las variables lámina de escorrentía (LE), lámina infiltrada (LI) y suelo erodado (SE) bajo el efecto de lluvia simulada a una intensidad de $15,5 \mathrm{~mm} / \mathrm{h}$, con periodos de 30 minutos en suelo disturbado.

\begin{tabular}{lcccc}
\hline \multirow{2}{*}{ FV } & \multirow{2}{*}{ GL } & \multicolumn{3}{c}{ CM } \\
\cline { 3 - 5 } & & LE (mm) & LI (mm) & SE (t.ha $\mathbf{~}^{-1}$ ) \\
\hline Uso & 3 & $58,699^{* *}$ & $74,798^{* *}$ & $0,859^{* *}$ \\
Pendiente & 2 & $93,865^{* *}$ & $60,303^{* *}$ & $1,401^{* *}$ \\
Uso*Pendiente & 6 & $10,103^{* *}$ & $10,620^{* *}$ & $0,211^{* *}$ \\
Tiempo & 5 & $10,162^{* *}$ & $11,489^{* *}$ & $0,024^{*}$ \\
Uso*Tiempo & 15 & $0,871^{*}$ & $0,896^{*}$ & $0,005 \mathrm{~ns}$ \\
Pendiente*Tiempo & 10 & $0,679^{*}$ & $0,668^{*}$ & $0,011^{*}$ \\
Uso*Pendiente*Tiempo & 30 & $0,186 \mathrm{~ns}$ & $0,204 \mathrm{~ns}$ & $0,004 \mathrm{~ns}$ \\
Error & 144 & & & \\
$\mathrm{r}^{2}$ & & 0,91 & 0,84 & 0,95 \\
\hline $\mathbf{C V}$ & & 24,85 & 14,05 & 11,69 \\
\hline
\end{tabular}

*: Diferencias significativas; $\mathrm{p}<0,05,{ }^{* *}$ : Diferencias altamente significativas; $\mathrm{p}<0,01, \mathrm{~ns}$ : Diferencias no significativas. 
Lámina de Escorrentía (LE). La prueba de Comparación de Medias de Fisher (Tabla 3), indica que el uso del suelo con bovinaza U2, presenta los valores más altos para lámina de escorrentía sugiriendo mayor remoción de las partículas superficiales del suelo, con 1,01; 2,51 y $4,96 \mathrm{~mm}$ a $10^{\circ}, 15^{\circ}$ y $25^{\circ}$ de pendiente respectivamente, en comparación al U4, que mostró un comportamiento más estable bajo el efecto de la lluvia simulada, con los valores más bajos de 0,40 y $0,61 \mathrm{~mm}$ a $15^{\circ}$ y $25^{\circ}$ de pendiente. Hay que destacar que conservan las tendencias para la pendiente de $10^{\circ}$ entre los usos estudiados.

Tabla 3. Comparación de Promedios (LSD) entre tratamientos para $10^{\circ}, 15^{\circ}$ y $25^{\circ}$ de pendiente, para Lámina de Escorrentía (mm).

\begin{tabular}{llccc}
\hline & \multirow{2}{*}{ Usos } & \multicolumn{3}{c}{ Grado de Pendiente } \\
\cline { 3 - 5 } & & $\mathbf{1 0}^{\circ}$ & $\mathbf{1 5}^{\circ}$ & $\mathbf{2 5}^{\circ}$ \\
\hline U2 & Bovinaza & $1,01 \mathrm{e}$ & $2,51 \mathrm{c}$ & $4,96 \mathrm{a}$ \\
U1 & Gallinaza & $0,91 \mathrm{fe}$ & $1,59 \mathrm{~d}$ & $3,57 \mathrm{~b}$ \\
U3 & Residuos Vegetales & $0,36 \mathrm{hg}$ & $1,13 \mathrm{e}$ & $2,30 \mathrm{c}$ \\
& Área de conservación & $0,15 \mathrm{~h}$ & $0,40 \mathrm{hg}$ & $0,61 \mathrm{fg}$ \\
\hline
\end{tabular}

Medias con la misma letra no son estadísticamente significativas.

Estos resultados, indican que posiblemente hay efectos negativos en la incorporación de abonos orgánicos en este suelo Pachic Fulvudands, pues se considera que tanto el uso, manejo y la calidad de estos, han acompañado un deterioro de la estabilidad estructural por la adición de estos abonos, la labranza y demás prácticas que desarrollan los agricultores. En este sentido, Malagón (1998) sugiere que la compactación, origina disminu- ción de la porosidad y como consecuencia, reducción de la infiltración, produciendo un encostramiento superficial y aumento en la escorrentía. Un comportamiento similar, se reportó en el cultivo de papa, en un estudio en el altiplano de Pasto, Nariño (Mena et al., 2011), utilizando suelos con historia de manejo con pastos, papa y bosque, encontrando lámina de escorrentía con valores bajos en el bosque y altos en el uso del suelo con pastos con $1,73 \mathrm{~mm}$ para el evento de lluvia simulada. Murray et al. (2014), indican que el desarrollo agrícola sustentable está en función del mantenimiento de la calidad del suelo, directamente relacionado con la susceptibilidad a la degradación que depende de la estabilidad estructural de los suelos.

En la interacción entre los factores uso, pendiente y tiempo para la variable lámina de escorrentía (Figura 5), se observa que a cada intervalo de tiempo y pendiente simulada, los usos, se ven influenciados directamente por efecto de la lluvia generada, donde el U2 presenta un comportamiento bastante significativo, el cual aumenta a medida que transcurre el tiempo y se incrementa el grado de pendiente. En comparación a U4 que mostró un comportamiento uniforme y leve ante los factores evaluados. Al respecto Greenland y Lal, (1977) señalan que es importante analizar la estabilidad de los agregados más superficiales, ya que por estar disponibles al impacto directo de las gotas de lluvia, son los más sensibles a romperse $\mathrm{y}$ formar una costra con sus constituyentes y por lo tanto, reducir la infiltración y aumentar la escorrentía. 


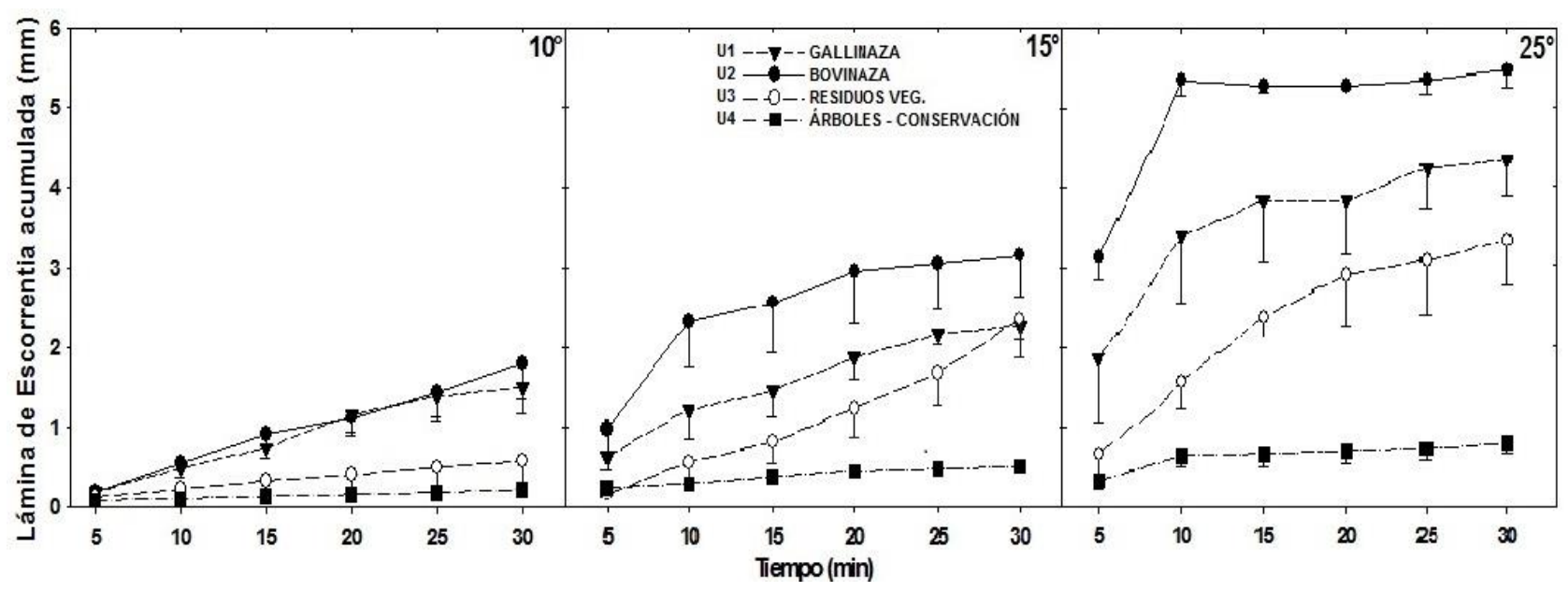

Figura 5. Promedios para lámina de escorrentía acumulada ( $\mathrm{mm}$ ) en lotes, con diferentes usos, con abonos orgánicos y área de conservación con árboles, mediante el uso de un minisimulador de lluvia, altiplano de Pasto, sur de Colombia.

Lámina de Infiltración (LI). En la Tabla 4 se presenta la comparación de medias de Fisher para la variable lámina de infiltración, indicando que el uso de suelo U4, muestra las tendencias con los valores más altos de infiltración para las tres pendientes $(7,18 ; 6,85 \mathrm{y}$ $6,97 \mathrm{~mm}$ ) respectivamente; sin embargo, para la pendiente de $10^{\circ}$ no se observan diferencias significativas de infiltración respecto al uso U3. El uso de suelo U2, presenta las tendencias con los valores promedios más bajos con 6,07; 4,60 y $2,32 \mathrm{~mm}$ sugiriendo suelos con menos capacidad de infiltración que se traduce en mayor riesgo de erosión. La influencia del grado de pendiente, determina la magnitud de procesos hidrológicos como la infiltración y la escorrentía superficial (Miyazaki, 1993).

Guerrero (1990) sostiene que la capacidad que tiene un suelo de permitir el paso de agua, se ve afectado solo si se mantiene un sistema de labranza por varios años, que permitan el cambio en la estructura del suelo a través de un número excesivo de labores o preparación del terreno. Los valores bajos de lámina infiltrada en un inceptisol con influencia volcánica, sugieren separabilidad de los suelos que originan sellado de la superficie (Castillo, 2004).

Tabla 4. Comparación de Promedios (LSD) entre tratamientos para $10^{\circ}$, $15^{\circ}$ y $25^{\circ}$ de pendiente, para Lámina de Infiltración (mm).

\begin{tabular}{llccc}
\hline & \multirow{2}{*}{ Usos } & \multicolumn{3}{c}{ Grado de Pendiente } \\
\cline { 3 - 5 } & & $\mathbf{1 0}^{\circ}$ & $\mathbf{1 5}^{\circ}$ & $\mathbf{2 5}^{\circ}$ \\
\hline U4 & Área de conservación con árboles & $7,18 \mathrm{a}$ & $6,85 \mathrm{ba}$ & $6,97 \mathrm{a}$ \\
U3 & Residuos Vegetales & $6,95 \mathrm{a}$ & $6,36 \mathrm{bc}$ & $5,70 \mathrm{de}$ \\
U1 & Gallinaza & $6,17 \mathrm{dc}$ & $5,51 \mathrm{e}$ & $4,08 \mathrm{f}$ \\
U2 & Bovinaza & $6,07 \mathrm{dc}$ & $4,60 \mathrm{f}$ & $2,32 \mathrm{~g}$ \\
\hline
\end{tabular}

Medias con la misma letra no son estadísticamente significativas. 
El análisis de los promedios de lámina de infiltración acumulada (Figura 6), muestra que el uso de suelo U4, presentó un comportamiento constante durante el tiempo de simulación de lluvia, presentando los valores promedio más altos de infiltración, mientras que el U2, presentó una disminución a medida que aumentó el tiempo de simulación de lluvia y pendientes, presentando los valores más bajos con respecto a los demás usos; la diferencia en los usos con manejo agrícola en comparación con el de conservación con árboles, puede ser debido a la estructura física, operaciones de labranza y la falta de cobertura, los cuales disminuyen en el largo plazo los macroporos y por ende la infiltración (Reid et al., 1990). Como efecto del comportamiento de la infiltración, la escorrentía aumentó progresivamente con la intensidad de uso del suelo a través del tiempo (Volverás et al., 2007).

Suelo erodado (SE). La prueba de comparación de medias de Fisher (Tabla 5), indica que el uso del suelo con bovinaza U2, presentó los valores más altos de suelo erodado con 0,072 t.ha- ${ }^{-1} ; 0,284$ t.ha ${ }^{-1}$ y 0,595 t.ha ${ }^{-1}$ a $10^{\circ}, 15^{\circ}$ y $25^{\circ}$ de pendiente, respectivamente, en comparación a U4, que mostró los valores promedios más bajos durante el tiempo de simula- ción, con valores de 0,008 t.ha ${ }^{-1} ; 0,029$ t.ha ${ }^{-1} \mathrm{y}$ 0,041 t.ha ${ }^{-1}$ de suelo erodado a las pendientes evaluadas. Los valores más altos de suelo erodado en U2, sugieren el efecto de la aplicación de bovinaza a largo plazo y posiblemente las inadecuadas prácticas de labranza aumentan la susceptibilidad a la erosión. León (2000) en su estudio, asocia los valores más altos de suelo erodado con la escaza cobertura y las deficientes prácticas de manejo del suelo que debilitan su estructura, aumentando la susceptibilidad a la erosión.

En la Figura 7, se observan los resultados para el análisis de promedios de suelo erodado acumulado (t.ha ${ }^{-1}$ ), que indican que U4 mantuvo un comportamiento constante durante los eventos en cada tiempo de simulación de lluvia y para cada pendiente, con valores promedios muy bajos de suelo erodado, comparados con bovinaza U2 que presentó los valores más altos para las simulaciones realizadas. Castillo (2008) en su estudio sobre la estimación de la estabilidad de suelos con diferentes tipos de manejo a largo plazo, concluye, que la actividad biológica inducida por la adición de abono orgánico (gallinaza) y coberturas de leguminosas, no afectó la estabilización de los agregados.

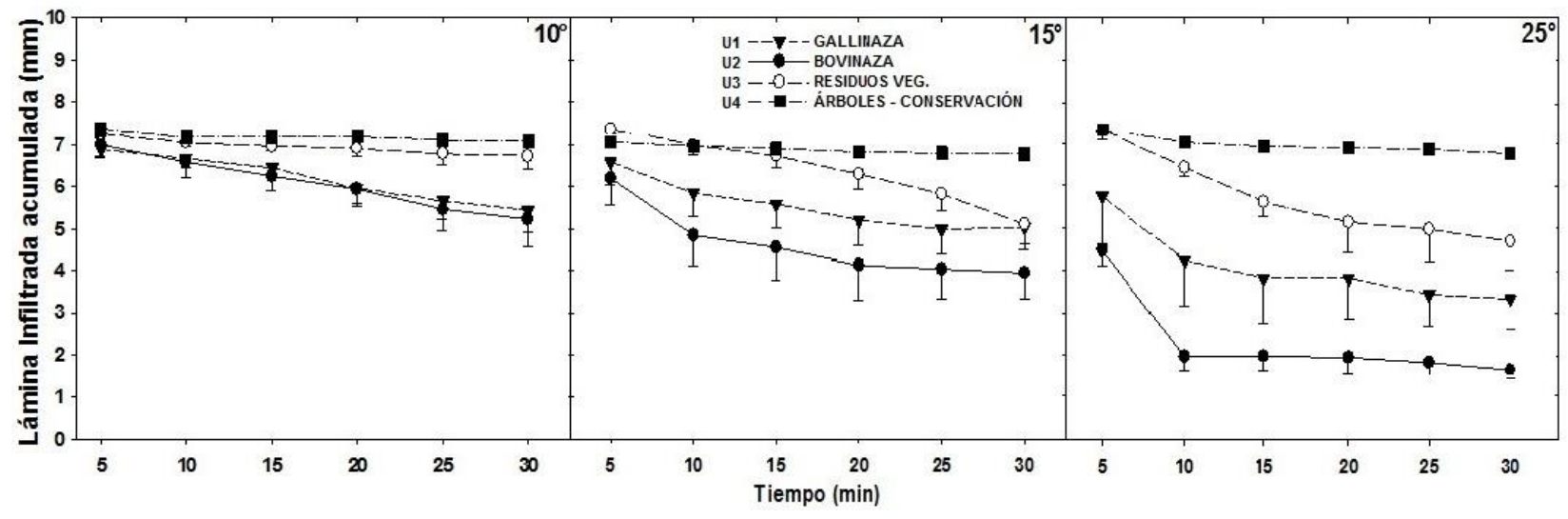

Figura 6. Promedios para Lámina de Infiltración Acumulada (mm) en lotes con diferentes usos con abonos orgánicos y área de conservación con árboles, mediante el uso de un minisimulador de lluvia, altiplano de Pasto, sur de Colombia. 
Tabla 5. Comparación de promedios (LSD) entre tratamientos para 10, 15 y $25^{\circ}$ de pendiente, para suelo erodado $\left(\mathrm{t} \cdot \mathrm{ha}^{-1}\right)$.

\begin{tabular}{llccc}
\hline & \multirow{2}{*}{ Usos } & \multicolumn{3}{c}{ Grado de Pendiente } \\
\cline { 3 - 5 } & & $\mathbf{1 0}^{\circ}$ & $\mathbf{1 5}^{\circ}$ & $\mathbf{2 5}^{\circ}$ \\
\hline U2 & Bovinaza & $0,072 \mathrm{fe}$ & $0,284 \mathrm{c}$ & $0,595 \mathrm{a}$ \\
U1 & Gallinaza & $0,035 \mathrm{fg}$ & $0,186 \mathrm{~d}$ & $0,407 \mathrm{~b}$ \\
U3 & Residuos Vegetales & $0,026 \mathrm{fg}$ & $0,087 \mathrm{e}$ & $0,206 \mathrm{~d}$ \\
U4 & Área de conservación con árboles & $0,008 \mathrm{~g}$ & $0,029 \mathrm{fg}$ & $0,041 \mathrm{feg}$ \\
\hline
\end{tabular}

Medias con la misma letra no son estadísticamente significativas.

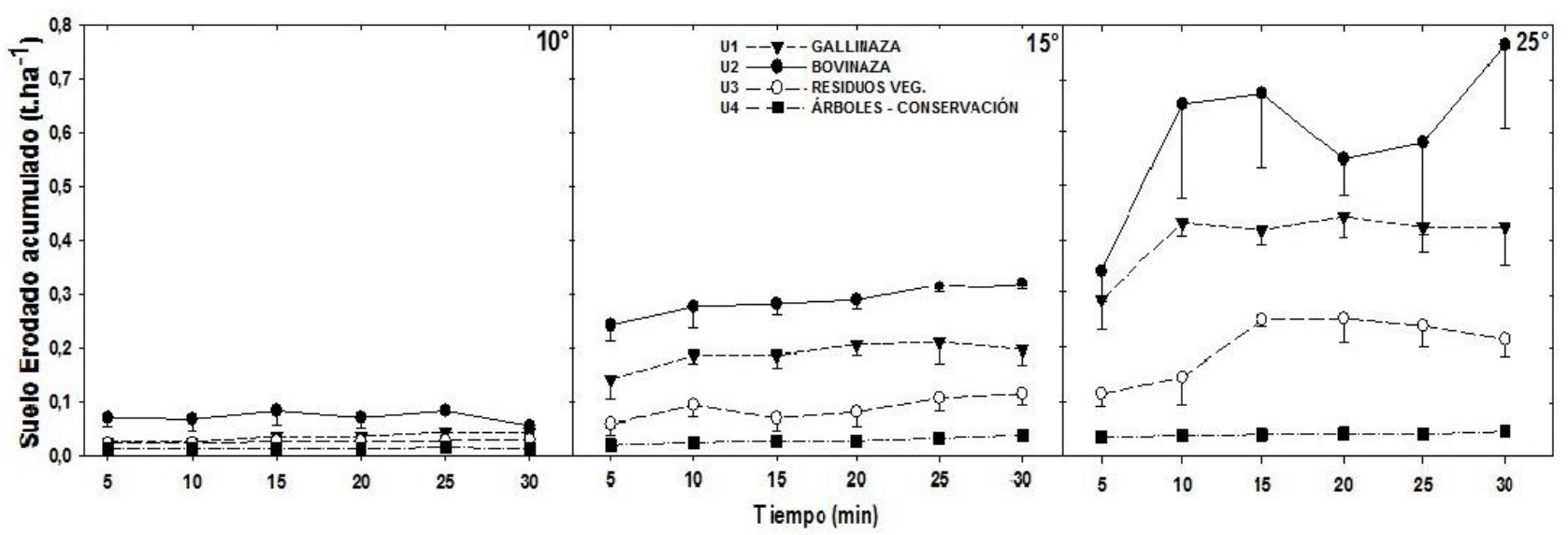

Figura 7. Separación de medias para la variable suelo erodado acumulado (t.ha-1), en lotes con diferentes usos con abonos orgánicos y área de conservación con árboles, mediante el uso de un minisimulador de lluvia, Altiplano de Pasto, sur de Colombia.

En general, se observa que el proceso erosivo, está muy influenciado por la capacidad de infiltración que tenga el suelo, la cual regula la escorrentía y ésta a su vez, está muy relacionada con la pérdida de suelo. Torres (2000), expresa que si la infiltración es alta, el suelo perdido será poco. La susceptibilidad del suelo a la escorrentía y a la erosión están estrechamente relacionadas con la estabilidad de los agregados de la superficie del suelo y la falta de continuidad de los poros del suelo (Pagliai et al., 2004).

Reid et al. (1990) estudió los cambios en el suelo, debido a la intervención agrícola y de- muestra como la agricultura reduce la capacidad de drenaje del suelo a largo plazo. Lo anterior sustenta las grandes diferencias encontradas en infiltración, escorrentía y erosión entre los usos, con intervención agrícola y el área de conservación con árboles, que se considera como poco intervenido.

Al analizar los resultados sobre infiltración, escorrentía y el suelo erodado en el tiempo, se deduce que hay una respuesta diferencial para los usos evaluados. El minisimulador de lluvia, permite identificar tales diferencias y la válida como una herramienta útil para la investigación sobre estudios de erosión. 
Respecto al DMP (Diámetro Medio Ponderado), el IGAC (2006) interpreta la estabilidad de agregados de la siguiente manera (Tabla 6).

Tabla 6. Diámetro Medio Ponderado de tamaño de agregados.

\begin{tabular}{ll}
\hline \multicolumn{1}{c}{ DMP (mm) } & \multicolumn{1}{c}{ Interpretación } \\
\hline$<0,5$ & Inestable \\
$0,5-1,5$ & Ligeramente Estable \\
$1,5-3,0$ & Moderadamente Estable \\
$3,0-5,0$ & Estable \\
$>5,0$ & Muy Estable \\
\hline
\end{tabular}

Considerando la interpretación anterior, en la Tabla 7, se observan los valores de DMP determinados para cada uso. Se observa que el U4 presenta el valor de DMP más alto que los demás usos, clasificándolo como muy estable, a diferencia del U2 y U1 que presentan los valores de DMP más bajos, clasificándolos como ligeramente estable y moderadamente estable, respectivamente según clasificación del IGAC, (2006).

Para el índice de estabilidad, el Uso U4 y U3 obtuvieron valores inferiores a uno. Esto indica que el suelo posee buena estabilidad y es más fácil recuperarse, frente a perturbaciones, especialmente a las provocadas por el agua. Para este caso, los usos mencionados anteriormente, poseen una buena estabilidad estructural a diferencia del U2 y U1 que obtuvieron valores superiores a 1 , los cuales indican mala estabilidad.

Al respecto, se infiere, que posiblemente el efecto de los árboles sobre el área de conservación, ha permitido que este suelo haya recuperado a través de los años, la estructura física y de igual manera la actividad biológica del mismo, viéndose reflejado en el comportamiento de este uso de suelo bajo la influencia de la lluvia simulada en el presente trabajo. Al respecto Muschler (1999), manifiesta que el efecto de los árboles a través del suelo (Mantenimiento de la fertilidad y estructura del suelo) tiene un impacto marcado, solamente después de años o décadas de sembrados, disminuyendo la escorrentía superficial y por ende, las mayores tasas de erosión del suelo. A ocho años de implantado un sistema agroforestal, mostró un aumento del 85\% de la materia orgánica edáfica (Murray et al., 2014).

Sin embargo, para las parcelas con uso de abonos orgánicos, se evidenció un comportamiento inverso, donde tal vez la aplicación de éste tipo de enmiendas orgánicas a largo plazo, está ocasionando complicaciones en las propiedades físicas del suelo adicionado al mal manejo, el compostaje y las prácticas de labranza que el agricultor este efectuando.

Tabla 7. Diámetro medio ponderado (DMP) e índice de estabilidad (IE) de la distribución porcentual de agregados estables al agua de los diferentes tratamientos.

\begin{tabular}{cc|cccc}
\hline \multirow{2}{*}{ Usos } & $\begin{array}{c}\text { U1 } \\
\text { Gallinaza }\end{array}$ & $\begin{array}{c}\text { U2 } \\
\text { Bovinaza }\end{array}$ & $\begin{array}{c}\text { U3 } \\
\text { Res. } \\
\text { Vegetales }\end{array}$ & $\begin{array}{c}\text { U4 } \\
\text { Conservación } \\
\text { con Árboles }\end{array}$ \\
\hline ⿷ & DMP & 2,00 & 1,40 & 2,80 & 5,60 \\
& IE & 1,20 & 1,57 & 0,88 & 0,02 \\
\hline
\end{tabular}


Sin embargo, en estudios de corto plazo (Cardona et al., 2016) encontraron que suelos cultivados que recibieron fertilizantes minerales y químicos, presentaron una estructura deficiente en contraste con suelos que recibieron aplicación de compost asociado con micorrizas. Acevedo et al. (2001) en su estudio sobre la estabilidad de agregados en seco y húmedo al evaluar el efecto de especies vegetales y abonos orgánicos, encontraron que la macroestructuración estuvo influenciada más por las especies vegetales que por los abonos orgánicos.

En éste sentido, adicionalmente malas prácticas de labranza en los suelos agrícolas conducen a la remoción, destrucción y transformación de los agregados naturales del suelo a tamaños menores, que son fácilmente dispersados por las gotas de lluvia y arrastrados por las aguas de escorrentía.

\section{CONCLUSIONES}

Los resultados obtenidos a través de las modificaciones estructurales y de diseño del minisimulador de lluvia, sugieren que el equipo puede ser utilizado en condiciones controladas de laboratorio y resulta una técnica que tiene validez para identificar diferencias por manejo en los suelos.

El uso del suelo con coberturas arbóreas de conservación (U4), presentaron la mayor lámina de infiltración, la menor lámina de escorrentía y los menores valores de suelo erodado, a las pendientes $10^{\circ}, 15^{\circ}$ y $25^{\circ}$, respectivamente.

El uso de suelo con aportes de bovinaza (U2), presentaron los valores más altos de lámina de escorrentía y suelo erodado a las pendientes de $10^{\circ}, 15^{\circ}$ y $25^{\circ}$, en su orden.
Los valores obtenidos para el Diámetro Medio Ponderado (DMP) y el Índice de Estructura (IE), indican que el uso con coberturas arbóreas de conservación (U4), posee una buena estabilidad, en comparación con bovinaza (U1) y gallinaza (U2), los cuales son más susceptibles a riesgos por erosión hídrica.

Los resultados sugieren, que la adición de enmiendas orgánicas acompañados de manejos inadecuados en el largo plazo, no son suficientes para disminuir riesgos por degradación física del suelo.

\section{REFERENCIAS BIBLIOGRÁFICAS}

1. Acevedo, S. O.; Velázquez, R. A.; Flores, R. D. 2001. Agregación por especies vegetales y abonos orgánicos en tepetates fracturados en condiciones de invernadero. Terra Latinoamericana. 19(4):363 - 373.

2. Alvarado, N. K.; López, P. V.; Castillo, J. A. 2011. Pérdida de suelo por erosión hídrica en diferentes sistemas de producción con papa Solanum tuberosum L. Colombia. Revista de Ciencias Agrícolas. 28(1):64 - 72.

3. Amézquita, E. 1994. Las propiedades físicas y el manejo productivo de los suelos. Inc: Silva, F. (ed), Fertilidad de suelos, diagnóstico y control. Sociedad Colombiana de la Ciencia del Suelo. Santafé de Bogotá, Colombia. 137 - 154p.

4. Beer, J.; Muschler, R.; Kass, D.; SOMARRIBA, E. 1998. Shade management in coffee and cacao plantations. Agrofor. Syst. 38:139 - 164.

5. Cardona, W. A.; Bolaños, B. M.; Chavarriaga, M. 2016. Efecto de fertilizantes químicos y orgánicos sobre la agregación de un suelo cultivado con Musa acuminata. Acta Agronómica. 65(2):144 - 148. 
6. Castillo, J. 2004. Variación de la erodabilidad y aplicación de la ecuación universal de pérdida de suelo (USLE) en los Andes colombianos. Universidad Nacional de Colombia, Palmira. Colombia. 165p.

7. Castillo, J. 2008. Estimación de estabilidad estructural de suelos del sur de Colombia con diferentes tipos de manejo. Acta Agronómica. 57(1):31 - 34.

8. Cobo, L. 1998. Diseño, construcción y evaluación de un mini-simulador portátil de lluvia para estudios de susceptibilidad a la erosión en laderas. Universidad del Valle - Universidad Nacional sede Palmira, Colombia. 64p.

9. Figueroa, S. B.; Amante, O. A.; Cortés, H. G.; Pimentel, L.; Osuna, C. J.; Rodríguez, E. S.; y Morales, F. J. 1991. Manual de predicción de pérdidas de suelo por erosión. SARHColegio de Postgraduados. Salinas, S.L.P., México. 150p.

10. Flanagan, D. C.; Nearing, M. A. 1995. Overview of the WEPP erosion prediction model. Technical documentation WEPP, Report No. 10. National Soil Erosion Research Laboratory. USDAARS-MWA. West Lafayette, Indican, USA. 3p.

11. Fraume, N. 2007. Diccionario ambiental. Edita Eco Ediciones. Colombia. 465p.

12. Greenland, D.; Lal, R. 1977. Soil conservation and management in the humid tropics. Soil Science. 126(1):61p.

13. Guerrero, L. 1990. Las propiedades físicas de los suelos colombianos y su relación con labores de labranza. pp697 - 728. En: Propiedades físicas de los suelos. Bogotá, Colombia, IGAC.

14. Holdridge, L. R. 2000. Ecología basada en Zonas de Vida. Quinta reimpresión. San José: Instituto Interamericano de Cooperación para la Agricultura. 216p.

15. IGAC - Instituto Geográfico Agustín Codazzi. 2004. Estudio general de suelos y zonificación de tierras del departamento de Nariño. IGAC. Tomo III. 72p.
16. IGAC - Instituto Geográfico Agustín Codazzi. 2006. Métodos analíticos del Laboratorio de Suelos. Bogotá D.C. Republica de Colombia. 407 - 410p.

17. IGAC - Instituto Geográfico Agustín Codazzi. 1995. Suelos de Colombia. Subdirección Agrológica. Santa Fé de Bogotá. 501 - 502p.

18. IDEAM - Instituto de Hidrología, Meteorología y Estudios Ambientales. 2012. Propuesta para la Gestión Integral Ambiental del Recurso Suelo (GIARS). Convenio interadministrativo de Asociación entre el Ministerio de Ambiente, Vivienda y Desarrollo Territorial y el Instituto de Hidrologia, Meteorologia y Estudios Ambientales. IDEAM. Bogotá. 79p.

19. IDEAM - Instituto de Hidrología, Meteorología y Estudios Ambientales. 2010. Pasto Nariño. Boletín divulgativo de información climatológica anual. IDEAM, Bogotá. 22p.

20. Lal, R. 1998. Agronomic consequences of soil erosion. pp.149 - 160. In: Penning De Vries, F.W.T.; Agus, f.; Kerr, J. Eds. Soil erosion at multiple scales. Principles and methods for assessing causes and impacts. CABI Publishing and IBSRAM.

21. León, T. S. 2000. Efectos de plantaciones forestales sobre suelo y agua. CONIF - Minambiente. Santafé de Bogotá. 5 - 84p.

22. Malagón, D. 1998. El Recurso Suelo en Colombia. Inventario y Problemática. Revista Académica Colombiana de la Ciencia de los Suelos. 22(82):13 - 52.

23. Mena, H. D.; Benavides, C.; Castillo, J. A. 2011. Evaluación de la susceptibilidad a la erosión hídrica de un vitric haplustands, mediante el uso de un minisimulador de lluvia, en una zona de ladera en Colombia. Revista de Ciencias Agrícolas. 28(2):70 - 80 .

24. Mendivelso, D.; Rubiano, Y.; Malagón, C. D.; López, L. D. 1998. Erosión de las tierras colombianas. En: Conferencias Magistrales: Manejo de Suelos e Impacto Ambiental. IX Congreso Colombiano de la Ciencia del Suelo. Paipa. 41 -49p. 
25. Miyazaki, T. 1993. Water flow in soils. Marcel Dekker, Inc. New York. 296p.

26. Murray, R. M.; Orozco, M. G.; Hernández, A.; Lemus, C.; Nájera, O. 2014. El sistema agroforestal modifica el contenido de materia orgánica y las propiedades físicas del suelo. Avances en Investigación Agropecuaria. 18(1):23 - 31.

27. Muschler, R. 1999. Módulo 5. Producción orgánica y agroforestal de cultivos perennes (café). CATIE. 59p

28. Oades, J.M.; Waters, A.G. 1991.Aggregate hierarchy in soils. Aust. J. Soil Res. 29(6):815 - 828.

29. Oldeman, L. R.; Hakkeling, R. T.; Sombroek, W. G. 1990. World map of the status of human-induced soil degradation. An explanatory note. ISRIC/UNEP, Wageningen. 35p.

30. Pagliai, M.; Vignozzi, N.; Pellegrini, A. 2004. Soil structure and the effect of management practices. Soil Tillage Research. 79:131 - 143.

31. Pimentel, D. 2006. Soil erosion: a food and environmental threat. Environment, development and sustainability. 8(1):119 137.

32. Quiroz, M. T.; Hincapié, G. E. 2007. Pérdidas de suelo por erosión en sistemas de producción de café con cultivos intercalados. CENICAFÉ. 58(3):227 - 235.

33. Reid, I.; Parkinson, R. J.; Twomlow, S. J.; Clark, A. 1990. The impact of agricultural land use changes on soil conditions and drainage. Vegetation and erosion Processes and environments. 199 - 215p.

34. Rivera, R. P.; Oropeza, J. L.; Martínez, M. R.; Mejía, S. E.; Tapia, L. M.; Ventura, R. JR. 2012. El proceso Lluvia-EscurrimientoErosión en laderas y microcuencas instrumentadas. Tecnología y Ciencias del Agua, vol. III, núm. 4, octubre-diciembre. Instituto Mexicano de Tecnología del Agua. Morelos, México. 151 - 166p.
35. Sánchez, G.; Pinzón, H.; Clímaco, J.; Herrera, C.A.; Martínez, E. P.; Quevedo, D. H.; Murcia, G. A.; Pedraza, R. A.; Martínez, P.; Ortíz, L. S.; Montaña, C. E.; Valderrama, Y.; Pinzón, L. M.; Rodríguez, J. 2012. Manual de Cebolla de Rama. Mosquera, Cundinamarca: Corpoica - Produmedios. 29 - 31p.

36. Torres, 2000. Evaluación de la Susceptibilidad a la Erosión y de las Características Asociadas (Infiltración y Escorrentía) de dos Suelos de Ladera en el Departamento del Cauca, Mediante un Minisimulador de lluvia. Universidad Nacional de Colombia, Cali, Valle. 9p.

37. Volverás, B.; Amézquita, E.; Táfur, H. 2007. Efecto del Tiempo del Uso en las Condiciones Físicas del Suelo Andino en el Departamento de Nariño. Acta Agronómica. 56(1):29 - 36. 